\title{
Spinal stenosis in familial transthyretin amyloidosis
}

AS Carr ${ }^{1}$, MRB Evans ${ }^{1}$, AL Pelayo-Negro ${ }^{1,2}$, S Shah ${ }^{3}$, D Choi $^{4}$, J Blake ${ }^{1,5}$, R Phadke $^{6}$, J Gilbertson ${ }^{7}$ CJ Whelan ${ }^{7}$, A D Wechalekar ${ }^{7}$, JD Gillmore ${ }^{7}$, PN Hawkins ${ }^{7}$, MM Reilly $^{1}$

1. MRC Centre for Neuromuscular Diseases, UCL Institute of Neurology and National Hospital for Neurology and Neurosurgery, Queen Square, London, UK.

2. Service of Neurology, University Hospital "Marqués de Valdecilla (IDIVAL)", University of Cantabria (UC) and "Centro de Investigación Biomédica en Red de Enfermedades Neurodegenerativas (CIBERNED)", Santander, Spain

3. Department of Neuroradiology, National Hospital of Neurology and Neurosurgery, Queen Square, London, UK.

4. Department of Neurosurgery, National Hospital of Neurology and Neurosurgery, Queen Square, London, UK.

5. Department of Clinical Neurophysiology, Norfolk and Norwich University Hospital, Norwich

$$
\text { UK. }
$$

6. Department of Neuropathology, National Hospital of Neurology and Neurosurgery, Queen Square, London, UK.

7. National Amyloidosis Centre, Royal Free Hospital, Rowland Hill Street, London, NW3 2PF, UK.

Word count: 1010

Figure: 1

\section{References: 11}

Corresponding author: Professor Mary M Reilly MD FRCP FRCPI

Professor of Clinical Neurology and Consultant Neurologist

Head of Division of Clinical Neurology, Institute of Neurology

MRC Centre for Neuromuscular Diseases

National Hospital for Neurology and Neurosurgery and UCL Institute of Neurology

Box 108, Queen Square, London WC1N 3BG

Phone: 0044 (0)845 1555000 Ext 88457

Fax: $0044(0) 2034483633$

E-mail:m.reilly@ucl.ac.uk 
Dear Professor Cornblath,

Systemic amyloidosis is a disorder of protein folding in which there is extracellular deposition of insoluble fibrillar proteins (Falk et al., 1997). At least 30 proteins are known to form amyloid deposits in vivo in humans and classification is based on the identity of the respective amyloid fibril precursor protein (Sipe et al., 2012). Transthyretin-associated amyloidosis (ATTR) is the commonest cause of hereditary amyloidosis, with more than 100 mutations in TTR that increase its amyloid-forming potential. Peripheral and autonomic neuropathy and cardiomyopathy are hallmarks of the disease but broad clinical heterogeneity is recognised (Plante-Bordeneuve and Said, 2011). Wild-type TTR is amyloidogenic in the very elderly with histological evidence of amyloid deposition in up to $25 \%$ of autopsied patients greater than 80 years old (Lie and Hammond., 1998). A restrictive cardiomyopathy is the best characterised manifestation with preceding carpal tunnel syndrome (CTS) in 48.9\%; the role of joint and ligament amyloid deposition in the pathogenesis of a variety of orthopaedic disorders in increasing (Pinney et al., 2013).

Lumbar spinal stenosis is typically presents in late middle age (Katz and Harris, 2008). It is characterised by compression of sensory and motor nerves to the lower limbs, exacerbated by exercise and relieved with rest; disability due to pain, impaired mobility and sphincter disturbance accrues with time (Suri et al., 2010). It is most often multifactorial; related to disc degeneration, spondylolisthesis and age-related degenerative processes affecting connective tissue. Amyloid deposits have been found in the ligamentum flavum of 25/26 lumbar canal stenosis cases, with co-localised immunohistochemical staining for transthyretin in 5/15 specimens studied (Westmark et al., 2014). In a larger orthopaedic 
cohort of 111 patients congo-red staining identified amyloid deposits in $42.6 \%$ with confirmation TTR-derived amyloid by Western blot or mass spectrometry in the majority of these: 39/44 (Sueyoshi et al., 2011). No definite cases of spinal stenosis due to mutant TTR have been described. Currently, surgical decompression is the mainstay of treatment with clinical improvement in most cases (Sasaji et al., 2011) but with recent developments in the treatment of systemic amyloidoses, particularly transthyretin-derived amyloidosis (Adams et la., 2016\}), should we be paying more attention to the underlying pathogenesis of this common condition?

Here we describe a patient with genetically confirmed ATTR, a family history of the disease and histological confirmation following carpal tunnel release surgery but no other manifestations. Finding ATTR deposits contributing to lumbar spinal stenosis had significant treatment implications.

A woman of German ancestry was found to be a carrier of a heterozygous 184S TTR mutation at the age of 42 . Genetic testing was performed on the basis of a strong family history of systemic amyloidosis affecting her mother, maternal uncle and older brother with carpal tunnel syndrome, peripheral neuropathy and restrictive cardiomyopathy. From this point she was under co-ordinated 2 yearly reviews by the National Amyloidosis Centre (NAC) in the Royal Free Hospital and peripheral nerve and autonomic services in the National Hospital of Neurology and Neurosurgery. At age 44, tissue confirmation was made on flexor retinaculum from carpal tunnel release surgery. She had been suffering from bilateral worsening CTS for approximately 10 years but denied symptoms of peripheral neuropathy, autonomic dysfunction, cardiac failure or visual impairment. Systemic and neurological examinations were normal except for positive Tinnel's at the left wrist. Nerve conduction studies (NCS) were normal other than evidence of bilateral CTS and borderline 
sural sensory nerve action potentials ( $8 \mu \mathrm{V}$ on the right). Normal thermal thresholds suggest lack of marked small fibre dysfunction. Autonomic screening tests and 24 hour BP monitoring were normal. ECOG performance status was 1, echocardiogram was normal but DPD scintigraphy (Perugini grade 1) and cardiac MRI were in keeping with early cardiac amyloidosis, NT pro-BNP=16 pMol/L. She was commenced on Diflunisal 500mg. There was no evidence of disease progression either subjectively or objectively over the next 4 years.

At age 47, she developed a patch of numbness on the dorsum of her left foot and pain in her lower back which radiated to both anterior thighs and knees. Initially this came on after walking approximately one mile and would worsen if she continued walking but settle completely after 10-15 minutes rest. Over the next 12 months the sensory disturbance in her foot progressed proximally along the $L 5$ dermatome, pain worsened in severity and exercise tolerance declined to less than 100 yards. She also described a tingling sensation in the same distribution and occasional feelings of her "legs giving way". She had long-standing urge incontinence of urine but latterly developed a constant leak with urinary and fecal incontinence and worsening of all symptoms on bending and straining. There were no upper limb complaints or symptoms above the neck. Neurological examination now revealed decreased, but not absent, pinprick sensation from the left great toe and dorsum of the foot respecting the $L 5$ dermatome and slight asymmetry of ankle and knee jerks with reinforcement required on the left. Pulses were present and straight leg raising test was limited to $40^{\circ}$.

An MRI spinal cord showed multiple lumbosacral disc bulges, most marked at $L 2 / 3$, superimposed on constitutional narrowing of the lumbar vertebral canal due to ligamentum flavum hypertrophy and neurosurgical referral for spinal decompression was made (Figure 
1a). Visualisation of the stenosed segment during surgery allowed for estimation of the relative contribution from the $L 2 / 3$ disc prolapse and ligamentum flavum hypertrophy as $30 \%$ and $70 \%$ respectively. Histological examination confirmed the presence of amyloid deposits, immunohistochemistry labelled these as transthyretin (Figure 1b). Six weeks postoperatively she reported marked improvement in pain, exercise tolerance was greater than one mile, continence had improved and the area of decreased sensation was reduced to a few $\mathrm{cm}^{2}$ around the dorsum of the left great toe, the rest of the neurological examination was normal.

This is the first reported case of mutant ATTR-associated spinal stenosis. Given overlapping ages of presentation of these conditions and the suggestion that TTR-amyloid may have a predilection for early deposition in connective tissue we suggest histological examination for amyloid should be considered after spinal decompression when this is caused by predominant ligamenum flavum hypertrophy and TTR gene analysis if this is positive. New therapeutic strategies in ATTR are emerging so early diagnosis may allow the individual to access disease modifying treatment and the broader family the benefit of genetic screening. 
TTR-spinal stenosis

\section{Acknowledgements, competing interests and funding}

M. M. R. is grateful to the Medical Research Council (MRC) and NINDS/ORD (1U54NS065712-01) for their support. This work was undertaken at University College London Hospitals/University College London, which received a proportion of funding from the Department of Health's National Institute for Health Research Biomedical Research Centres funding scheme. 


\section{Legend}

Figure 1a MRI lumbosacral spine. Sagittal T1 reveal moderate background degenerative disc bulges at $L 2 / 3, L 4 / 5$ and $L 5 / S 1$ superimposed on constitutional narrowing of thelumbar canal $(A)$, in combination with ligamentum flavum thickening causing moderate stenosis of the vertebral canal.

Figure 1b: Ligamentum flavum histology. A formalin-fixed, paraffin-embedded section from the ligamentum flavum biopsy ( $\mathrm{L} 2 / 3$ level) shows one of the fragments of the ligament rich in elastic fibres (arrow) containing several amorphous eosinophilic proteinaceous deposits (stars) (A). These deposits stain strongly with the Congo Red dye (arrow) (B) and are seen to extend in to the cartilaginous intervertebral disc (arrow) that shows severe degenerative changes (C). The Congo Red-positive deposits show characteristic apple-green birefringence when visualised under polarised light confirming their amyloid nature (D).The deposits stain strongly with TTR-specific immunoflourescent antibody (E, F). Scale bar in A,B,C, E = $100 \mu \mathrm{m}$ and $D, F=50 \mu \mathrm{m}$ 


\section{References}

1. Falk RH, Comenzo RL, Skinner M. The systemic amyloidosis. N Engl. J Med 1997;337:898-909.

2. Sipe JD, Benson MD, Buxbaum JN, Ikeda S, Merlini G, Saraiva MJ, et al. Amyloid fibril protein nomenclature: 2012 recommendations from the nomenclature committee of the International Society of AMyloidosis. Amyloid. 2012;19:167-170.

3. Plante-Bordeneuve V, Said G. Familial amyloid polyneuropathy. Lancet Neurol 2011;10:108697.

4. Lie JT, Hammond PI. Pathology of the senescent heart: Anatomic observations on 237 autopsy studies of patient 95-105 years old. Mayo Clin Proc 1998;63:552-564.

5. Pinney JH, Whelan CJ, Petrie A, Dungu J, Banypersad SM, et al. Sensile systemic amyloidosis: clinical features at presentation and outcome. J Am Heart Assoc. 2013;2:e000098.

6. Katz JN, Harris MB. Lumbar spinal stenosis. N Engl J Med 2008;358:818-825.

7. Suri $P$, Rainville J, Kalichman L, Katz JN. Does this older individual with lower extremity pain have the syndrome of lumbar canal stenosis? JAMA. 2010;304:2628-36.

8. Westermark P, Westermark GT, Suhr OB, Berg S. Transthyretin-derived amyloidosis: probably a common cause of lumbar spinal stenosis. Ups J Med Sci 2014;119:223-228.

9. Sueyoshi T, Ueda M, Jono H, Irie H, Sei A, Ide J, Ando Y, Mizuta H. Wild-type transthyretinderived amyloidosis in various ligaments and tendons. Human Pathology 2011;42:12591264.

10. Sasaji T, Horaguchi K, Yamada N, Iwai K. Improvement of hip abductor muscle weakness after lumbar decompressive surgery. Ups J Med Sci 2012;117:426-9.

11. Adams D, Cauquil C, Labeyrie C, Beaudonnet G, Algalarrondo V, Théaudin M .TTR kinetic stabilizers and TTR gene silencing: a new era in therapy for familial amyloidotic polyneuropathies. Expert Opin Pharmacother. 2016;17(6):791-802. 